University of Wollongong

Research Online

Faculty of Engineering and Information

Faculty of Engineering and Information

Sciences - Papers: Part B

Sciences

2019

Explicit finite difference analysis of an unsteady magnetohydrodynamics heat and mass transfer micropolar fluid flow in the presence of radiation and chemical reaction through a vertical porous plate

R Biswas

Khulna University

M Mondal

Khulna University

Kazi Shanchia

Khulna University

Rubel Ahmed

University of Wollongong, ra972@uowmail.edu.au

S Samad

Khulna University

See next page for additional authors

Follow this and additional works at: https://ro.uow.edu.au/eispapers1

Part of the Engineering Commons, and the Science and Technology Studies Commons

Research Online is the open access institutional repository for the University of Wollongong. For further information contact the UOW Library: research-pubs@uow.edu.au 


\title{
Explicit finite difference analysis of an unsteady magnetohydrodynamics heat and mass transfer micropolar fluid flow in the presence of radiation and chemical reaction through a vertical porous plate
}

\begin{abstract}
The investigation of this paper is undertaken to study of the problem of chemical reaction on MHD unsteady heat and mass transfer effects of micropolar fluid flow through a vertical plate. Different effects such as thermal diffusion, Soret and Dufour effects in the presence of radiation and chemical reaction are permitted in this observation. Firstly, the governing partial differential equations (PDEs) are transformed into a system of dimensionless coupled partial differential equations by the procedure of mathematical transformation. Then the obtained dimensionless equations are solved by explicit finite difference method (EFDM). Further, the momentum, temperature and concentration distributions are permitted by the variations of various parameters with the help of computer programming language FORTRAN. By using initial boundary conditions $\mathrm{U}=0, \mathrm{~V}=0, \Gamma=0, T=1, \mathrm{C}=1$ and for $\Delta \tau=0.0005, \Delta \mathrm{X}=0.83$ and $\Delta \mathrm{Y}=$ 0.50 , the convergence criteria are exhibited $\mathrm{Pr} \geq 0.205$ and $\mathrm{S}_{\mathrm{c}} \geq 0.045$. In addition, the streamlines and isotherms are evaluated for different interesting parameters. Finally, after stability convergence test (SCT) the profiles of velocity, angular velocity, temperature and concentration are analysed and scrutinized graphically by using graphs software tacplot- 9 .

\section{Disciplines}

Engineering | Science and Technology Studies

\section{Publication Details}

Biswas, R., Mondal, M., Shanchia, K., Ahmed, R., Samad, S. Abdus. \& Ahmmed, S. F. (2019). Explicit finite difference analysis of an unsteady magnetohydrodynamics heat and mass transfer micropolar fluid flow in the presence of radiation and chemical reaction through a vertical porous plate. Journal of Nanofluids, 8 (7), 1583-1591.
\end{abstract}

\section{Authors}

R Biswas, M Mondal, Kazi Shanchia, Rubel Ahmed, S Samad, and S Ahmmed 

ca

Explicit Finite Difference Analysis of an Unsteady Magnetohydrodynamics Heat and Mass Transfer Micropolar Fluid Flow in the Presence of Radiation and Chemical Reaction Through a Vertical Porous Plate

\author{
R. Biswas ${ }^{1, *}$, M. Mondal', Kazi Shanchia', R. Ahmed², SK. Abdus Samad', and S. F. Ahmmed' \\ ${ }^{1}$ Mathematics Discipline, Science, Engineering and Technology School, Khulna University, Khulna-9208, Bangladesh \\ ${ }^{2}$ Faculty of Engineering and Information Sciences, University of Wollongong, NSW-2522, Australia
}

\begin{abstract}
The investigation of this paper is undertaken to study of the problem of chemical reaction on MHD unsteady heat and mass transfer effects of micropolar fluid flow through a vertical plate. Different effects such as thermal diffusion, Soret and Dufour effects in the presence of radiation and chemical reaction are permitted in this observation. Firstly, the governing partial differential equations (PDEs) are transformed into a system of dimensionless coupled partial differential equations by the procedure of mathematical transformation. Then the obtained dimensionless equations are solved by explicit finite difference method (EFDM). Further, the momentum, temperature and concentration distributions are permitted by the variations of various parameters with the help of computer programming language FORTRAN. By using initial boundary conditions $U=0, V=0$, $\bar{\Gamma}=0, \bar{T}=1, \bar{C}=1$ and for $\Delta \tau=0.0005, \Delta X=0.83$ and $\Delta Y=0.50$, the convergence criteria are exhibited $P_{r} \geq 0.205$ and $S_{c} \geq 0.045$. In addition, the streamlines and isotherms are evaluated for different interesting parameters. Finally, after stability convergence test (SCT) the profiles of velocity, angular velocity, temperature and concentration are analysed and scrutinized graphically by using graphs software tacplot-9.
\end{abstract}

KEYWORDS: Free Convection, MHD Flow, Micropolar Fluid, Chemical Reaction, Porous Medium.

\section{INTRODUCTION}

Now a days, micropolar fluids has been become a great interesting research area of fluid mechanics because the materials flow are highly used in the industrial and medical science as well as elsewhere. Micropolar fluids are those types of fluids which are contains micro-constituents as like as nanofluid. Also, micropolar fluids can be undergo with rotation and which can be affected by the magnetohydrodynamics (MHD) of the fluid flow. Furthermore, the behaviour of micropolar fluids are likely as non-Newtonian which consisting of dumb-bell molecules, polymer fluids and fluid suspension. There are many applications of micropolar fluids, such as analysing the behaviour of polymeric fluids, additive suspensions and liquid crystals. Also, human and animal blood, turbulent shear flow, exotic lubricants, the flow of colloidal suspensions are having due to

\footnotetext{
Author to whom correspondence should be addressed.

Email: rajibkumath11@gmail.com

Received: 4 Novenber 2018

Accepted: 14 December 2018
}

micropolar fluids. In 1996, the theory of micropolar fluids was first introduced by Eringen. ${ }^{1}$ Haque et al. ${ }^{2}$ have introduced the behaviours of micropolar fluid on steady MHD free convection flow with constant heat and mass fluxes, joule heating and viscous dissipation. From their study, it is observed that the momentum, angular momentum, temperature and concentration equations was solved by shouting method with similarity technique. Also, self-similar solution of incompressible micropolar boundary layer flow was scrutinized by Ahmadi. ${ }^{3}$ Ariman $^{4}$ have focused the different applications of molecular fluid mechanics. Steady free convection heat and mass transfer MHD flow of a radiated micropolar fluid was reported by Nayak et al. ${ }^{5}$ Also, Balram and Sastry ${ }^{6}$ have carried out the micropolar free convection flow. Analysis of free convection micropolar boundary layer about a horizontal permeable cylinder has been capitalized by Lien. ${ }^{7}$ Tripathy et al. ${ }^{8}$ have discussed the numerical analysis MHD micropolar fluid. Flow of Micropolar fluid over an off cantered rotating disk with modified Darcy's law was submitted by 
Khan et al. ${ }^{9}$ Thermophoretic particle deposition on MHD flow of micropolar fluid is addressed by Doh et al. ${ }^{10}$ and energy transfer through mixed convection within square enclosure containing micropolar fluid was introduced by Muhammad et al. ${ }^{11}$

Study of the effects of heat and mass transfer on fluids flowboth Newtonian and non-Newtonian has become an important part in the last few years. This is so much importance in engineering applications and many industrial processes which experiences not only temperature difference but also concentration difference. However, it also used in many industrial applications and engineering devices. Peddieson ${ }^{12}$ has introduced the boundary layer theory for a micropolar fluid. Mass transfer and heat generation effects on MHD free convection flow past an inclined vertical surface in a porous medium was constructed by Reddy et al. ${ }^{13}$ Sparrow et al. ${ }^{14}$ have premeditated the effect of a magnetic field on free convection heat transfer. Ganesan et al. ${ }^{15}$ have adopted the natural convection effects on impulsively started inclined plate. Also, Islam et al. ${ }^{16}$ have analysed the Dufour and Soret effects on steady MHD free convection and mass transfer fluid. Finite difference analysis of unsteady natural convection MHD flow past an inclined plate with variable surface was discussed by Ganesan et al. ${ }^{17}$

In many mass transfer processes and heat transfer considerations are raised by chemical reaction and which are often due to the nature of the process. In this processes, such as drying, evaporation at the surface water body, energy transfer in a wet cooling tower and the flow in a desert cooler, heat and mass transfer occur simultaneously. During such chemical reactions, there is always generating of heat. The most common fluid fluids like water and air are contaminated with impurities like $\mathrm{CO}_{2}, \mathrm{C}_{6} \mathrm{H}_{6}$ and $\mathrm{H}_{2} \mathrm{SO}_{4}$. Chemical reaction parameter shows a retarding effect on concentration distribution as the reaction proceeds from constructive to destructive state. Bhuvaneswari et al. ${ }^{18}$ have explored the effects of radiation convective flow over an inclined plate in a porous medium. Mohanty et al.$^{19}$ have introduced the numerical investigation on heat and mass transfer effect of Micropolar fluid. Chemical reaction and Soret effects on Micropolar fluid flow was elaborated by Mishra et al. ${ }^{20}$ and MHD Flow with chemical reaction was capitalized by Rawat et al. ${ }^{21}$ Effects of radiation and chemical reaction on MHD was established by Biswas et al. ${ }^{22}$ Also, Rashidi et al. ${ }^{23}$ have probed the free convective heat and mass transfer for MHD fluid flow.

In the last few decades, the study of magnetohydrodynamics (MHD) mass transfer flow takes placed in many industrial applications such as polymer technology, aerodynamics heating, petroleum industry, electrostatic precipitation, power generators and petroleum industry. Effects of Hall current and chemical reaction on MHD unsteady heat and mass transfer of Casson nanofluid flow through a vertical plate was performed by Biswas et al.$^{24}$ Unsteady magnetohydrodynamics (MHD) free convection flow of nanofluid through an exponentially accelerated inclined plate embedded in a porous medium with variable thermal conductivity in the presence of radiation was recently published by Ahmmed et al. ${ }^{25}$ Mahanthesh et al. ${ }^{26}$ have found the unsteady three-dimensional MHD flow of a nano Eyring-Powell fluid in the effects of thermal radiation, viscous dissipation and Joule heating. Also, chemical reaction and radiated MHD heat and mass transfer flow with temperature dependent viscosity past an isothermal oscillating cylinder has been adopted by Ahmed et al. ${ }^{27}$ They have solved their governing equation by using explicit finite difference method. Further, Afikuzzaman et al. $^{28}$ have elaborated the unsteady MHD Casson fluid flow through a parallel plate with Hall current. Furthermore, MHD Casson fluid flow through a parallel plate was focused by Afikuzzaman and Alam. ${ }^{29}$ MHD free convective heat transfer in a Walter's liquid-B fluid past a convectively heated stretching sheet with partial wall slip was recently published by Seth et al., ${ }^{30}$ Farooq et al., ${ }^{31-32}$ Kumari et al., ${ }^{33}$ Jain et al.. ${ }^{34}$ Muhammad et al. ${ }^{35}$ and Shit et $\mathrm{al}^{36}$ have submitted the MHD flow of Eyring-Powell liquid in convectively curved configuration.

Motivated by all these above studies, a purpose is carried out to study the free convection Micropolar fluid flow on MHD unsteady heat and mass transfer in the presence of radiation and chemical reaction through a vertical porous plate. The obtained dimensionless governing partial differential equations (PDEs) is solved numerically by explicit finite difference method (EFDM) where FORTRAN programming language are used to find out numerical values. The fluid velocity, temperature and concentration profiles are shown graphically for the different values of different parameters. Also, the values of skin friction, Nusselt number and Sherwood number for the variation of different parameters are displayed in the form of table. At the end a comparison table has been shown.

\section{MATHEMATICAL ANALYSIS}

In this research work, $x$-axis is chosen along the plate where the fluid flow is accordingly and the $y$-axis is normal to the plate. Initially, it is considered that the temperature of the wall is assumed to be $T_{\mathrm{w}}$ and concentration at the wall is $C_{\mathrm{w}}$ in the fluid. At time $t>0$, fluid flow moves with a velocity $u$ then the concentration and temperature of the fluid are raised to $C_{\mathrm{w}}\left(=C_{\infty}\right)$ and $T_{\mathrm{w}}\left(=T_{\infty}\right)$. Further, it is assumed that a magnetic field $B_{y}=B_{0}$ of uniform strength is applied normal to the flow region. The physical configuration and coordinate system of the problem is presented in the following Figure 1.

Under the assumptions, the dimensional continuity, momentum, energy and concentration equations of micropolar fluid flow in the presence of chemical reaction, heat 


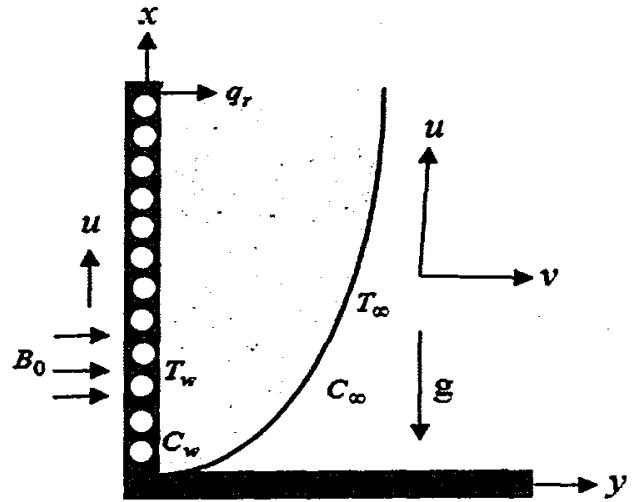

Fig. 1. Physical model and coordinate system.

generation and radiation subjected to the Boussinesq's approximation can be written in the following as

$$
\begin{aligned}
& \frac{\partial u}{\partial x}+\frac{\partial v}{\partial y}=0 \\
& \frac{\partial u}{\partial t}+u \frac{\partial u}{\partial x}+v \frac{\partial u}{\partial y}=\left(v+\frac{\chi}{\rho}\right) \frac{\partial^{2} u}{\partial y^{2}}+\frac{\chi}{\rho} \frac{\partial \Gamma}{\partial y} \\
& +g \beta_{\mathrm{T}}\left(T-T_{\infty}\right)+g \beta_{\mathrm{C}}\left(C-C_{\infty}\right) \\
& -\frac{v}{k^{*}} u \\
& \frac{\partial \Gamma}{\partial t}+u \frac{\partial \Gamma}{\partial x}+v \frac{\partial \Gamma}{\partial y}=\frac{\gamma}{\rho j} \frac{\partial^{2} \Gamma}{\partial y^{2}}-\frac{\chi}{\rho j} \frac{\partial u}{\partial y} \\
& \frac{\partial T}{\partial t}+u \frac{\partial T}{\partial x}+v \frac{\partial T}{\partial y}=\frac{k}{\rho C_{\mathrm{p}}} \frac{\partial^{2} T}{\partial y^{2}}-\frac{1}{\rho C_{\mathrm{p}}} \frac{\partial q_{\mathrm{r}}}{\partial y} \\
& +\frac{Q}{\rho C_{\mathrm{p}}}\left(T-T_{\infty}\right)+\frac{D_{\mathrm{m}} k_{\mathrm{T}}}{C_{\mathrm{S}} C_{\mathrm{P}}} \frac{\partial^{2} C}{\partial y^{2}} \\
& +\frac{\mu}{\rho C_{\mathrm{P}}}\left(\frac{\partial u}{\partial y}\right)^{2} \\
& \frac{\partial C}{\partial t}+u \frac{\partial C}{\partial x}+v \frac{\partial C}{\partial y}=D_{\mathrm{m}} \frac{\partial^{2} C}{\partial y^{2}}-K_{1}\left(C-C_{\infty}\right) \\
& +\frac{D_{\mathrm{m}} K_{\mathrm{T}}}{T_{\mathrm{m}}} \frac{\partial^{2} T}{\partial y^{2}}
\end{aligned}
$$

The associate initial and boundary conditions according to the present model are,

$$
\begin{aligned}
& u=0, \quad v=0, \quad \Gamma=0, \quad T=T_{\mathrm{w}}, \quad C=C_{\mathrm{w}} \quad \text { at } y=0 \\
& u=0, \quad v=0, \quad \Gamma=0, \quad T \rightarrow T_{\infty}, \quad C \rightarrow C_{\infty} \quad \text { as } y \rightarrow \infty
\end{aligned}
$$

Here, $u$ are $v$ are the velocity components in the $x$ and $y$-axis respectively, $\rho$ is the fluid density, $C_{\mathrm{p}}$ is the specific heat at constant pressure, $C_{\mathrm{s}}$ is concentration susceptibility, $k$ is the thermal conductivity, $v$ is the kinematic viscosity, $g$ is the gravitational acceleration, $k_{\mathrm{T}}$ is the thermal diffusion ratio, $T$ is the temperature, $C$ is the concentration component of the fluid, $\beta_{\mathrm{T}}$ is the thermal expansion coefficient, $\beta_{c}$ is the concentration expansion coefficient, $\Gamma$ is the microrotational component, $q_{\mathrm{r}}$ is the radiated heat flux, $k^{*}$ is the Darcy permeability, $T_{\mathrm{m}}$ is the mean fluid temperature, $q_{\mathrm{r}}$ is the radiated heat flux, $\chi$ is the vortex viscosity and $j$ is the micro inertia per unit mass.

The following dimensionless variables are consider:

$$
\begin{gathered}
U=\frac{u}{U_{0}} ; \quad V=\frac{v}{U_{0}} ; \quad \Gamma=\bar{\Gamma} \frac{U_{0}^{2}}{v} ; \quad Y=\frac{y U_{0}}{v} ; \\
X=\frac{x U_{0}}{v} ; \quad \tau=\frac{t U_{0}^{2}}{v} \\
T=T_{\infty}+\bar{T}\left(T_{\mathrm{w}}-T_{\infty}\right) ; \quad C=C_{\infty}+\bar{C}\left(C_{\mathrm{w}}-C_{\infty}\right)
\end{gathered}
$$

By using this above dimensionless variables, the following nonlinear coupled partial differential equations are obtained:

$$
\begin{gathered}
\frac{\partial U}{\partial X}+\frac{\partial V}{\partial Y}=0 \\
\frac{\partial U}{\partial \tau}+U \frac{\partial U}{\partial X}+V \frac{\partial U}{\partial Y}=(1+M P) \frac{\partial^{2} U}{\partial Y^{2}}+M P \frac{\partial \bar{\Gamma}}{\partial Y} \\
+G_{\mathrm{r}} \bar{T}+G_{\mathrm{m}} \bar{C}-K p U \\
\frac{\partial \bar{\Gamma}}{\partial \tau}+U \frac{\partial \bar{\Gamma}}{\partial X}+V \frac{\partial \bar{\Gamma}}{\partial Y}=S G \frac{\partial^{2} \bar{\Gamma}}{\partial Y^{2}}-V P \frac{\partial U}{\partial Y} \\
\frac{\partial \bar{T}}{\partial \tau}+U \frac{\partial \bar{T}}{\partial X}+V \frac{\partial \bar{T}}{\partial Y}=\frac{1}{P_{\mathrm{r}}}\left(1+\frac{4}{3} R_{\mathrm{a}}\right) \frac{\partial^{2} \bar{T}}{\partial Y^{2}}+S \bar{T} \\
+D_{\mathrm{u}} \frac{\partial^{2} \bar{C}}{\partial Y^{2}}+E_{\mathrm{c}}\left(\frac{\partial U}{\partial Y}\right)^{2} \\
\frac{\partial \bar{C}}{\partial \tau}+U \frac{\partial \bar{C}}{\partial X}+V \frac{\partial \bar{C}}{\partial Y}=\frac{1}{S_{\mathrm{c}}} \frac{\partial^{2} \bar{C}}{\partial Y^{2}}-G a \bar{C}+S_{\mathrm{r}} \frac{\partial^{2} \bar{T}}{\partial Y^{2}}
\end{gathered}
$$

Also, the associate boundary conditions are,

$$
\left.\begin{array}{c}
U=0, \quad V=0, \quad \bar{\Gamma}=0, \quad \bar{T}=1, \quad \bar{C}=1 \quad \text { at } Y=0 \\
U=0, \quad V=0, \quad \bar{\Gamma}=0, \quad \bar{T} \rightarrow 0, \quad \bar{C} \rightarrow 0 \quad \text { as } Y \rightarrow \infty
\end{array}\right\}
$$

where, $U, \bar{\Gamma}, \bar{T}$ and $\bar{C}$ represents the dimensionless velocity, angular velocity, fluid temperature and fluid concentration respectively. Also, $\tau$ is the time constant, $S_{\mathrm{c}}=$ $v / D_{\mathrm{m}}$ is Schmidt number, $G_{\mathrm{a}}=K_{1} v / U_{0}^{2}$ is chemical reaction parameter, $E_{\mathrm{c}}=U_{0}^{2} / C_{\mathrm{p}}\left(T_{\mathrm{w}}-T_{\infty}\right)$ is Eckert number, $P_{\mathrm{r}}=\rho C_{\mathrm{p}} v / k$ is Prandlt number, $G_{\mathrm{r}}=v g B_{\mathrm{T}}\left(T_{\mathrm{w}}-T_{\infty}\right) / U_{0}^{3}$ is Grashof number, $G_{\mathrm{m}}=v g B_{\mathrm{c}}\left(C_{\mathrm{w}}-C_{\infty}\right) / U_{0}^{3}$ is modified Grashof number, $R_{\mathrm{a}}=4 \sigma^{\prime} T_{\infty}^{3} / k k^{\prime}$ is radiation parameter, $S=Q v /\left(\rho C_{\mathrm{p}} U_{0}^{2}\right)$ is heat source parameter, $K_{\mathrm{p}}=v^{2} / k^{*} U_{0}^{2}$ is permeability of porous medium, $D_{\mathrm{u}}=\left(D_{\mathrm{m}} K_{\mathrm{T}}\left(C_{\mathrm{w}}-\right.\right.$ $\left.\left.C_{\infty}\right)\right) /\left(C_{\mathrm{s}} C_{\mathrm{p}} v\right)\left(T_{\mathrm{w}}-T_{\infty}\right)$ is Dufour Number, $M P=\chi / \rho v$ is microrotational parameter, $V P=\chi / \rho J U_{0}$ is vortex viscosity parameter, $S G=\gamma / \nu \rho j$ is spin gradient viscosity parameter and $S_{\mathrm{r}}=\left(D_{\mathrm{m}} K_{\mathrm{T}}\left(T_{\mathrm{w}}-T_{\infty}\right)\right) / T_{\mathrm{m}} v\left(C_{\mathrm{w}}-C_{\infty}\right)$ is Soret number.

Non-dimensional quantities skin friction coefficient, Nusselt number, and Sherwood number are carried out respectively by the following equations as

$$
C_{\mathrm{f}}=-\frac{1}{2 \sqrt{2}}\left(G_{\mathrm{r}}\right)^{-3 / 4}\left(\frac{\partial U}{\partial Y}\right)_{Y=0}
$$




$$
\begin{aligned}
& N_{\mathrm{u}}=\frac{1}{\sqrt{2}}\left(G_{\mathrm{r}}\right)^{-3 / 4}\left(\frac{\partial \bar{T}}{\partial Y}\right)_{Y=0} ; \\
& S_{\mathrm{h}}=\frac{1}{\sqrt{2}}\left(G_{\mathrm{r}}\right)^{-3 / 4}\left(\frac{\partial \bar{C}}{\partial Y}\right)_{Y=0}
\end{aligned}
$$

Stream function $\psi(X, Y)$ represents the following expressions with the velocity components as:

$$
U=\frac{\partial \psi}{\partial Y}, \quad V=-\frac{\partial \psi}{\partial X}
$$

\section{CALCULATION TECHNIQUE}

The finite difference method is a numerical and computer based method. For simplicity, the explicit finite difference method (EFDM) has been used to solve the Eqs. (9) to (13). To obtain a set of finite difference equations, the region within boundary layer of the flow are divided into a grid of lines parallel to $X$ and $Y$ axis, where $X$ axis is taken along the plate and $Y$ axis is normal to the plate which are depicted in the Figure 2.

Here, it is considered that the plate of height $X_{\max }$ $(=125)$ i.e., $X$ varies from 0 to 125 and regard $Y_{\max }(=150)$ i.e., $Y$ varies from 0 to 150 . The number of grid spacing are $m=150$ and $n=300$ along in the $X$ and $Y$ directions respectively. Also, It is assumed that $\Delta X, \Delta Y$ are constant mesh sizes along $X$ and $Y$ directions respectively and taken as $\Delta X=0.83(0 \leq X \leq 125)$ and $\Delta Y=0.50(0 \leq X \leq 150)$ with the smaller time-step, $\Delta \tau=0.0005$.

Now $U^{\prime}, \bar{\Gamma}^{\prime}, \bar{T}^{\prime}$ and $\bar{C}^{\prime}$ denote the values of $U, \bar{\Gamma}, \bar{T}$ and $\bar{C}$ at the end of a time-step respectively. Using the explicit finite difference approximation, the following appropriate set of finite difference relations are obtained,

$$
\begin{gathered}
\frac{U_{\mathrm{i}, \mathrm{j}}-U_{\mathrm{i}-1, \mathrm{j}}}{\Delta X}+\frac{V_{\mathrm{i}, \mathrm{j}}-V_{\mathrm{i}, \mathrm{j}-1}}{\Delta Y}=0 \\
\frac{U_{\mathrm{i}, \mathrm{j}}^{\prime}-U_{\mathrm{i}, \mathrm{j}}}{\Delta \tau}+U_{\mathrm{i}, \mathrm{j}}\left(\frac{U_{\mathrm{i}, \mathrm{j}}-U_{\mathrm{i}-1, \mathrm{j}}}{\Delta X}\right)+V_{\mathrm{i}, \mathrm{j}}\left(\frac{U_{\mathrm{i}, \mathrm{j}+1}-U_{\mathrm{i}, \mathrm{j}}}{\Delta Y}\right)
\end{gathered}
$$

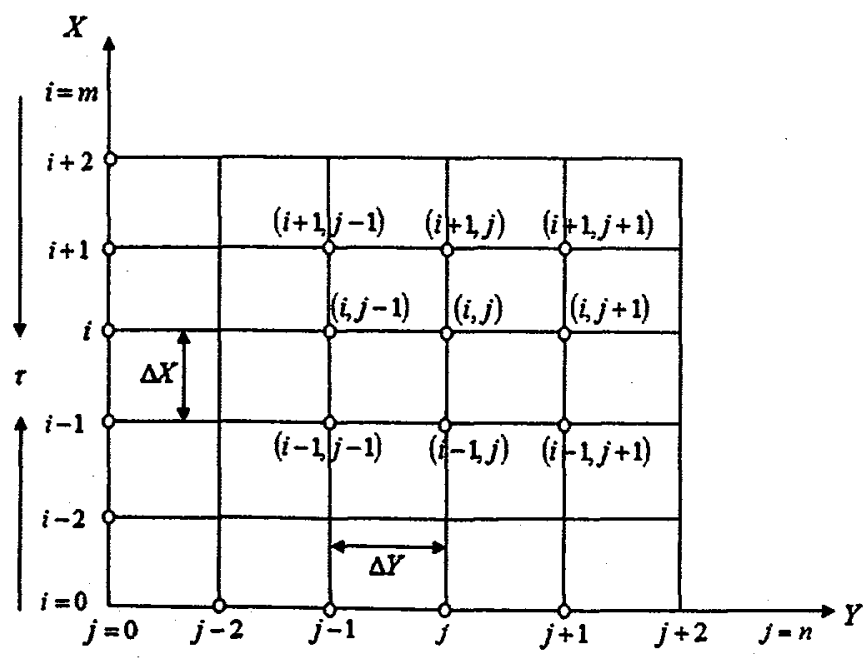

Fig. 2. The finite difference space grid.

$$
\begin{aligned}
& =(1+M P)\left(\frac{U_{\mathrm{i}, \mathrm{j}+1}-2 U_{\mathrm{i}, \mathrm{j}}+U_{\mathrm{i}, \mathrm{j}-1}}{(\Delta Y)^{2}}\right)+G_{\mathrm{r}} \bar{T}_{\mathrm{i}, \mathrm{j}} \\
& +G_{\mathrm{m}} \bar{C}_{\mathrm{i}, \mathrm{j}}+M P \frac{\bar{\Gamma}_{\mathrm{i}, \mathrm{j}+1}-\bar{\Gamma}_{\mathrm{i}, \mathrm{j}}}{\Delta Y}-k_{\mathrm{p}} U_{\mathrm{i}, \mathrm{j}}
\end{aligned}
$$

$$
\begin{aligned}
& \frac{\bar{\Gamma}_{\mathrm{i}, \mathrm{j}}^{\prime}-\bar{\Gamma}_{\mathrm{i}, \mathrm{j}}}{\Delta \tau}+U_{\mathrm{i}, \mathrm{j}}\left(\frac{\bar{\Gamma}_{\mathrm{i}, \mathrm{j}}-\bar{\Gamma}_{\mathrm{i}-1, \mathrm{j}}}{\Delta X}\right)+V_{\mathrm{i}, \mathrm{j}}\left(\frac{\bar{\Gamma}_{\mathrm{i}, \mathrm{j}+1}-\bar{\Gamma}_{\mathrm{i}, \mathrm{j}}}{\Delta Y}\right) \\
& =S G\left(\frac{\bar{\Gamma}_{\mathrm{i}, \mathrm{j}+1}-2 \bar{\Gamma}_{\mathrm{i}, \mathrm{j}}+\bar{\Gamma}_{\mathrm{i}, \mathrm{j}-1}}{(\Delta Y)^{2}}\right)-V P\left(\frac{U_{\mathrm{i}, \mathrm{j}+1}-U_{\mathrm{i}, \mathrm{j}}}{\Delta Y}\right)
\end{aligned}
$$

$$
\begin{aligned}
& \frac{\bar{T}_{\mathrm{i}, \mathrm{j}}^{\prime}-\bar{T}_{\mathrm{i}, \mathrm{j}}}{\Delta \tau}+U_{\mathrm{i}, \mathrm{j}} \frac{\bar{T}_{\mathrm{i}, \mathrm{j}}-\bar{T}_{\mathrm{i}-\mathrm{i}, \mathrm{j}}}{\Delta X}+V_{\mathrm{i}, \mathrm{j}} \frac{\bar{T}_{\mathrm{i}, \mathrm{j}+1}-\bar{T}_{\mathrm{i}, \mathrm{j}}}{\Delta Y} \\
& =\frac{1}{P_{\mathrm{r}}}\left(1+\frac{4}{3} R_{\mathrm{a}}\right) \frac{\bar{T}_{\mathrm{i}, \mathrm{j}+1}-2 \bar{T}_{\mathrm{i}, \mathrm{j}}+\bar{T}_{\mathrm{i}, \mathrm{j}-1}}{(\Delta Y)^{2}}+S \bar{T}_{\mathrm{i}, \mathrm{j}} \\
& \quad+D_{\mathrm{u}} \frac{\bar{C}_{\mathrm{i}, \mathrm{j}+1}-2 \bar{C}_{\mathrm{i}, \mathrm{j}}+\bar{C}_{\mathrm{i}, \mathrm{j}-1}}{(\Delta Y)^{2}}+E_{\mathrm{c}}\left(\frac{U_{\mathrm{i}, \mathrm{j}+1}-U_{\mathrm{i}, \mathrm{j}}}{\Delta Y}\right)^{2}
\end{aligned}
$$

$$
\begin{aligned}
& \frac{\bar{C}_{\mathrm{i}, \mathrm{j}}^{\prime}-C_{\mathrm{i}, \mathrm{j}}}{\Delta \tau}+U_{\mathrm{i}, \mathrm{j}} \frac{\bar{C}_{\mathrm{i}, \mathrm{j}}-\bar{C}_{\mathrm{i}-1, \mathrm{j}}}{\Delta X}+V_{\mathrm{i}, \mathrm{j}} \frac{\bar{C}_{\mathrm{i}, \mathrm{j}+1}-\bar{C}_{\mathrm{i}, \mathrm{j}}}{\Delta Y} \\
& =\frac{1}{S_{\mathrm{c}}} \frac{\bar{C}_{\mathrm{i}, \mathrm{j}+1}-2 \bar{C}_{\mathrm{i}, \mathrm{j}}+\bar{C}_{\mathrm{i}, \mathrm{j}-1}}{(\Delta Y)^{2}}-G a \bar{C}_{\mathrm{i}, \mathrm{j}} \\
& \quad+S_{\mathrm{r}} \frac{\bar{T}_{\mathrm{i}, \mathrm{j}+1}-2 \bar{T}_{\mathrm{i}, \mathrm{j}}+\bar{T}_{\mathrm{i}, \mathrm{j}-1}}{(\Delta Y)^{2}}
\end{aligned}
$$

In this case, the associate boundary conditions are:

$$
\begin{array}{llll}
U_{i, 0}^{n}=0, & V_{i, 0}^{n}=0, & \bar{\Gamma}_{i, 0}^{n}=0, & \bar{T}_{i, 0}^{n}=1, \quad \bar{C}_{i, 0}^{n}=1 \\
U_{i, \mathrm{~L}}^{n}=0, & \bar{\Gamma}_{\mathrm{i}, \mathrm{L}}^{n}=0, & \bar{T}_{\mathrm{i}, \mathrm{L}}^{n} \rightarrow 0, & \bar{C}_{\mathrm{i}, \mathrm{L}}^{n} \rightarrow 0
\end{array}
$$

where $L \rightarrow \infty$

\section{STABILITY AND CONVERGENCE ANALYSIS}

The stability conditions for the present problem are,

$$
\begin{gathered}
(1+M P) \frac{2 \Delta \tau}{(\Delta Y)^{2}}+U \frac{\Delta \tau}{\Delta X}+|-V| \frac{\Delta \tau}{\Delta Y}+\frac{K_{\mathrm{p}} \Delta \tau}{2} \leq 1 \\
\frac{1}{P_{\mathrm{r}}}\left(1+\frac{4}{3} R_{\mathrm{a}}\right) \frac{2 \Delta \tau}{(\Delta Y)^{2}}+U \frac{\Delta \tau}{\Delta X}+|-V| \frac{\Delta \tau}{\Delta Y}-\frac{S \Delta \tau}{2} \leq 1 \\
\frac{1}{S_{\mathrm{c}}} \frac{2 \Delta \tau}{(\Delta Y)^{2}}+U \frac{\Delta \tau}{\Delta X}+|-V| \frac{\Delta \tau}{\Delta Y}+\frac{G a \Delta \tau}{2} \leq 1
\end{gathered}
$$

With the initial boundary conditions $U=0, V=0, \bar{\Gamma}=0$, $\bar{T}=1, \bar{C}=1$ at $\tau=0$ and for the values of $\Delta \tau=0.0005$, $\Delta X=0.83$ and $\Delta Y=0.50$, the problem will be converged at $P_{\mathrm{r}} \geq 0.205$ and $S_{\mathrm{c}} \geq 0.045$. These converge solutions are shown graphically in Figures 3-7. 

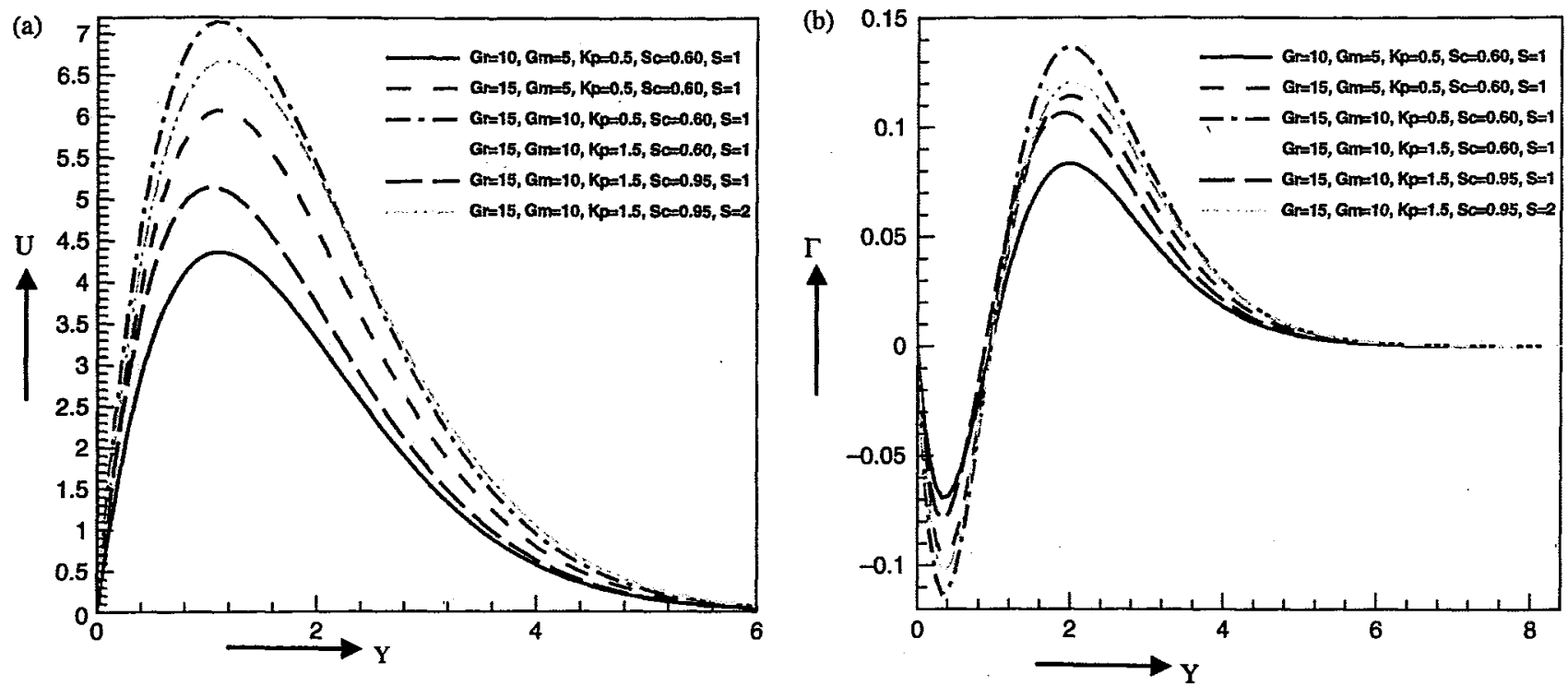

Fig. 3. Illustration of (a) velocity profiles and (b) angular velocity profiles for different values of $G_{r}, G_{m}, K_{p}, S_{c}$ and $S$.

\section{RESULTS AND DISCUSSION}

An explicit finite difference technique has been used to obtain the results of the present model and the approximate solutions are obtained for various parameters for the purpose of discussing the results of the problem. The physical situation of the present problem, the velocity, angular velocity, temperature, concentration, Skin friction, Nusselt number, Sherwood number, streamlines and isotherms for vertical plate within the boundary conditions are obtained for different values of radiation parameter $\left(R_{\mathrm{a}}\right)$, permeability of porous medium $\left(K_{\mathrm{p}}\right)$, Soret number $\left(S_{\mathrm{r}}\right)$, Eckert number $(E c)$, microrotational parameter $(M P)$, chemical reaction parameter $\left(G_{\mathrm{a}}\right)$, spin gradient viscosity parameter $(S G)$, vortex viscosity parameter $(V P)$, Schmidt number $\left(S_{\mathrm{c}}\right)$, heat source parameter $(S)$, Prandtl number $\left(P_{\mathrm{r}}\right)$,
Grashof number $\left(G_{\mathrm{r}}\right)$, modified Grashof number $\left(G_{\mathrm{m}}\right)$ and Dufour number $\left(D_{\mathrm{u}}\right)$ which are shown in Figures 3 to 7 . In order to obtain the accuracy of the numerical results, the following values of default parameter are chosen as: $P_{\mathrm{r}}=0.71, S_{\mathrm{c}}=0.60, R_{\mathrm{a}}=0.50, E_{\mathrm{c}}=0.01, D_{\mathrm{u}}=0.50, S=$ $1.0, S_{\mathrm{r}}=1, G_{\mathrm{m}}=5, G_{\mathrm{r}}=10, K_{\mathrm{p}}=1, S_{\mathrm{r}}=1, S G=0.5, V P=$ $0.1, M P=0.1$ and $G_{\mathrm{a}}=0.60$ with time $\tau=1$. These values are treated as common throughout the study in respective figures and tables.

The non-dimensional Grashof number $\left(G_{\mathrm{r}}\right)$, modified Grashof number $\left(G_{\mathrm{m}}\right)$, permeability of porous medium $\left(K_{\mathrm{p}}\right)$, Schmidt number $\left(S_{\mathrm{c}}\right)$ and heat source parameter $(S)$ on velocity and angular velocity are displayed in Figures 3(a) and (b) respectively. It is explored that the velocity profiles are increasing respectively as Grashof
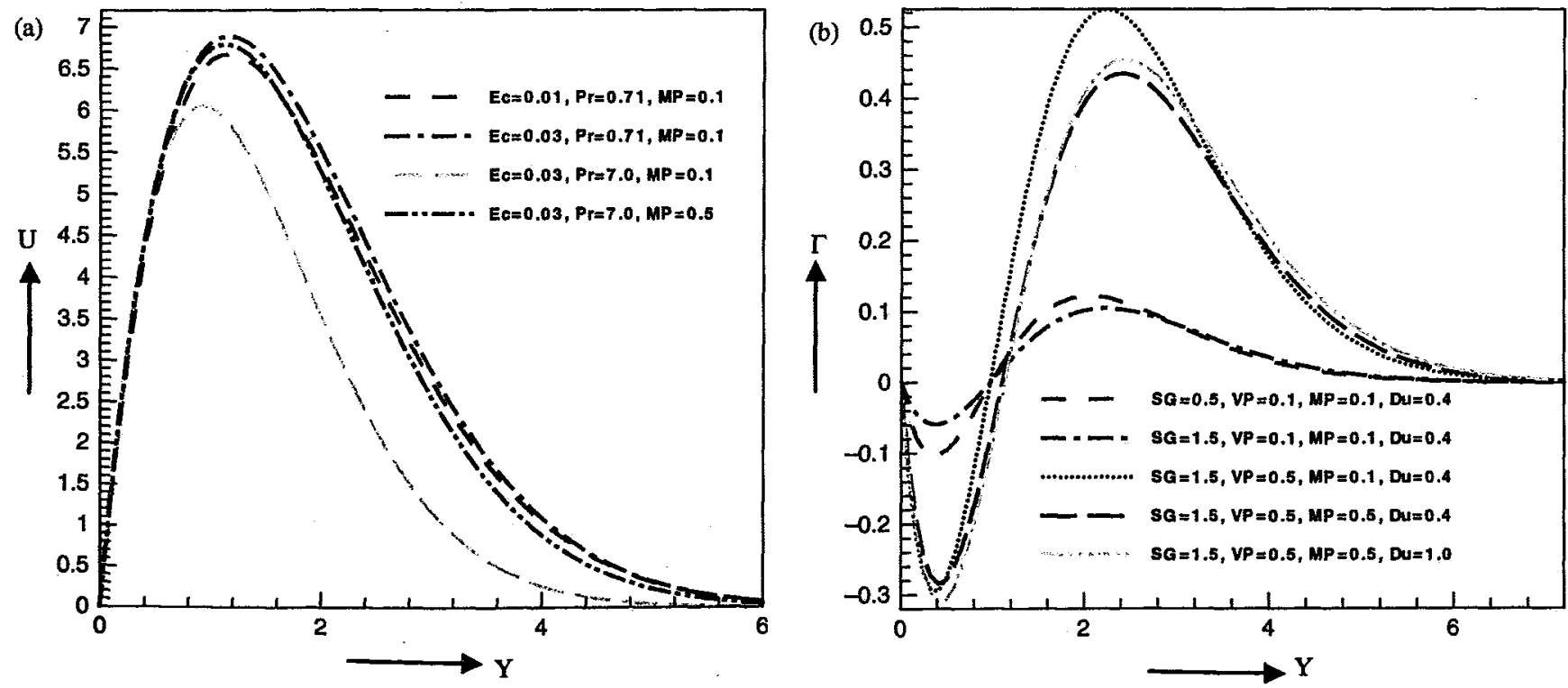

Fig. 4. Illustration of (a) velocity profiles for different values of $E_{\mathrm{c}}, P_{\mathrm{r}}$ and $M P$ and (b) angular velocity profiles of $S G, V P, M P$ and $D_{\mathrm{u}}$. 

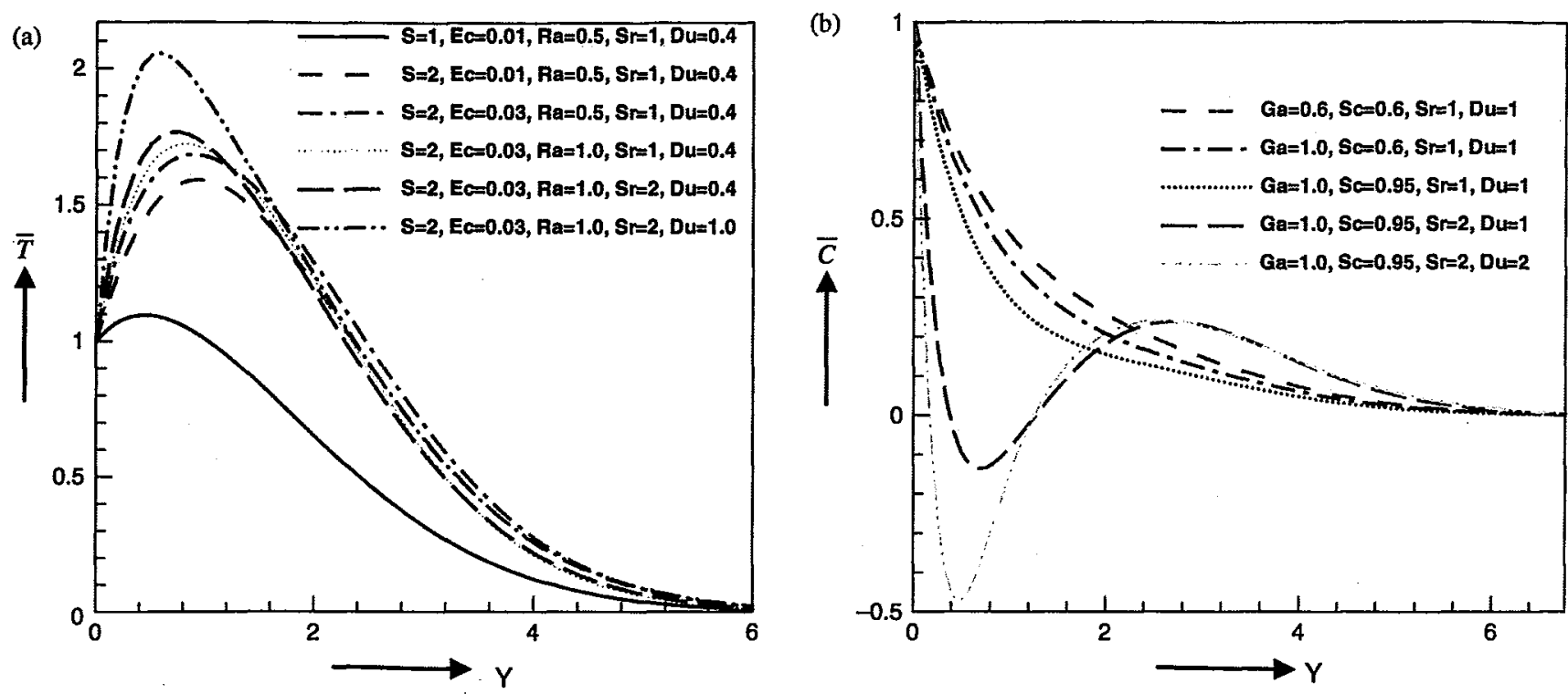

Fig. 5. Illustration of (a) temperature profiles for different values of $S, E_{\mathrm{c}}, R_{\mathrm{a}}, S_{\mathrm{r}}$ and $D_{\mathrm{u}}$ and (b) concentration profiles for different values of $G_{\mathrm{a}}, S_{\mathrm{c}}$, $S_{\mathrm{r}}$ and $D_{u}$.

number, modified Grashof number and heat source parameter but decreasing due to increase of permeability of porous medium and Schmidt number which are shown in Figure 3(a). Also, angular velocity profiles are increasing by the increase of Grashof number, Schmidt number, modified Grashof number and heat source parameter but just decreasing due to increase of permeability of porous medium. The thermal Grashof number which signifies the relative effect of the thermal buoyancy force in the boundary layer. Due to this augmentation of thermal buoyancy force which executing on the fluid particles for gravitational force that increase of the thickness of momentum boundary layer. This is the fact that velocity and angular velocity are increasing due to increase of Grashof number. Also a similar effect is visible in the vicinity of modified Grashof number.

For various values of Eckert number $(E c)$, Prandtl number $\left(P_{\mathrm{r}}\right)$ and microrotational parameter $(M P)$ on velocity profiles are delimitated in Figure 4(a). It is accomplished that velocity profiles are increasing for the increase of Eckert number and microrotational parameter but decrease due to the increase of Prandtl number. But from Figure 4(b), we have observed that angular velocity decreases $20.2 \%$ as spin gradient viscosity parameter changes to $S G=0.5$ to $S G=1.5$ but microrotational parameter increases $27.2 \%$ changes as $M P=0.1$ to $M P=0.5$ and increase $42.5 \%$ for $V P=0.1$ to $V P=0.5$. Also, angular velocity decreases $35.7 \%$ for changes as $D u=0.4$ to $D u=1.0$ which are shown in Figure 4(b).

The influence of heat source parameter $(S)$, Eckert number $(E c)$, radiation parameter $\left(R_{\mathrm{a}}\right)$, Soret number $\left(S_{\mathrm{r}}\right)$ and Dufour number $\left(D_{\mathrm{u}}\right)$ on temperature profiles are displaced in Figure 5(a). It is mentioned that the temperature of the fluid increased by $34.49 \%, 25.50 \%, 24.25 \%, 30.3 \%$ and $16.95 \%$ for changes as $S=1.0$ to $S=2.0, E_{\mathrm{c}}=0.01$ to $E_{\mathrm{c}}=$ $0.03, R_{\mathrm{a}}=0.50$ to $R_{\mathrm{a}}=1.0, S_{\mathrm{r}}=1.0$ to $S_{\mathrm{r}}=2.0$ and $D_{\mathrm{u}}=0.4$
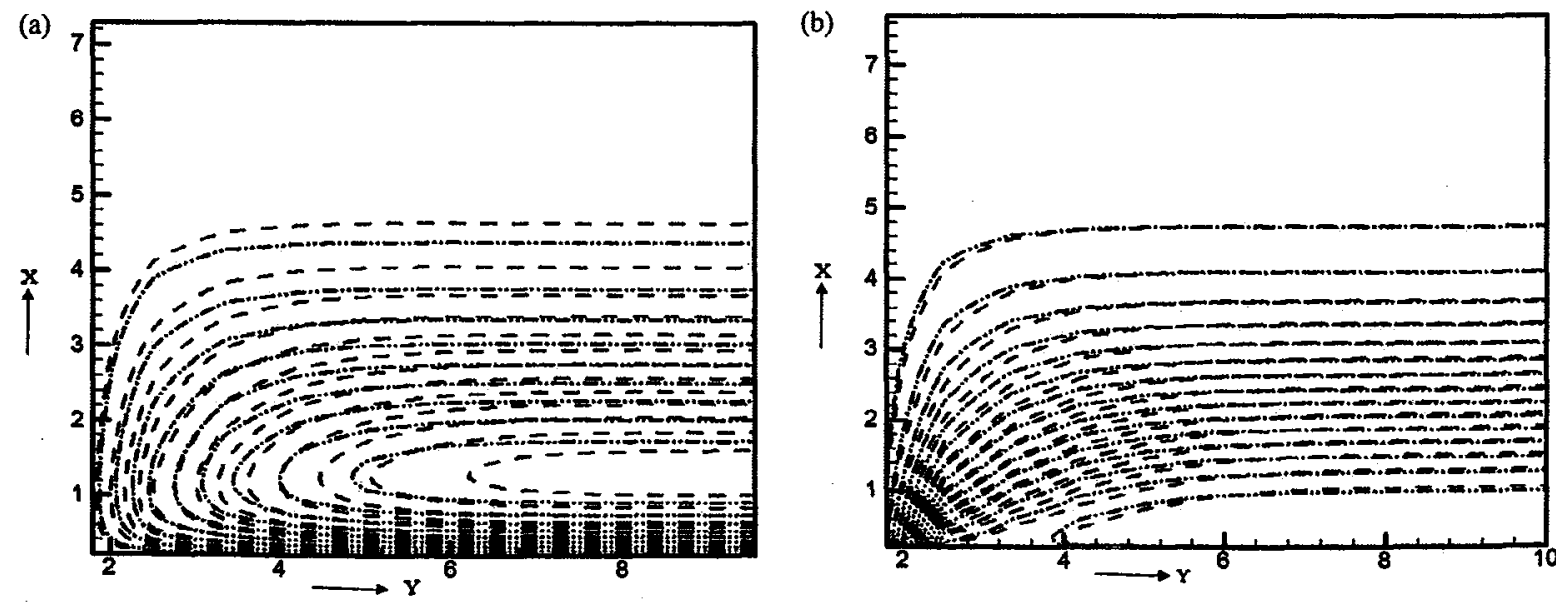

Fig. 6. Ilustration of (a) streamlines for $R_{\mathrm{a}}=0.50$ and $R_{\mathrm{a}}=1.50$ and (b) isotherms for $R_{\mathrm{a}}=0.50$ and $R_{\mathrm{a}}=1.50$. 

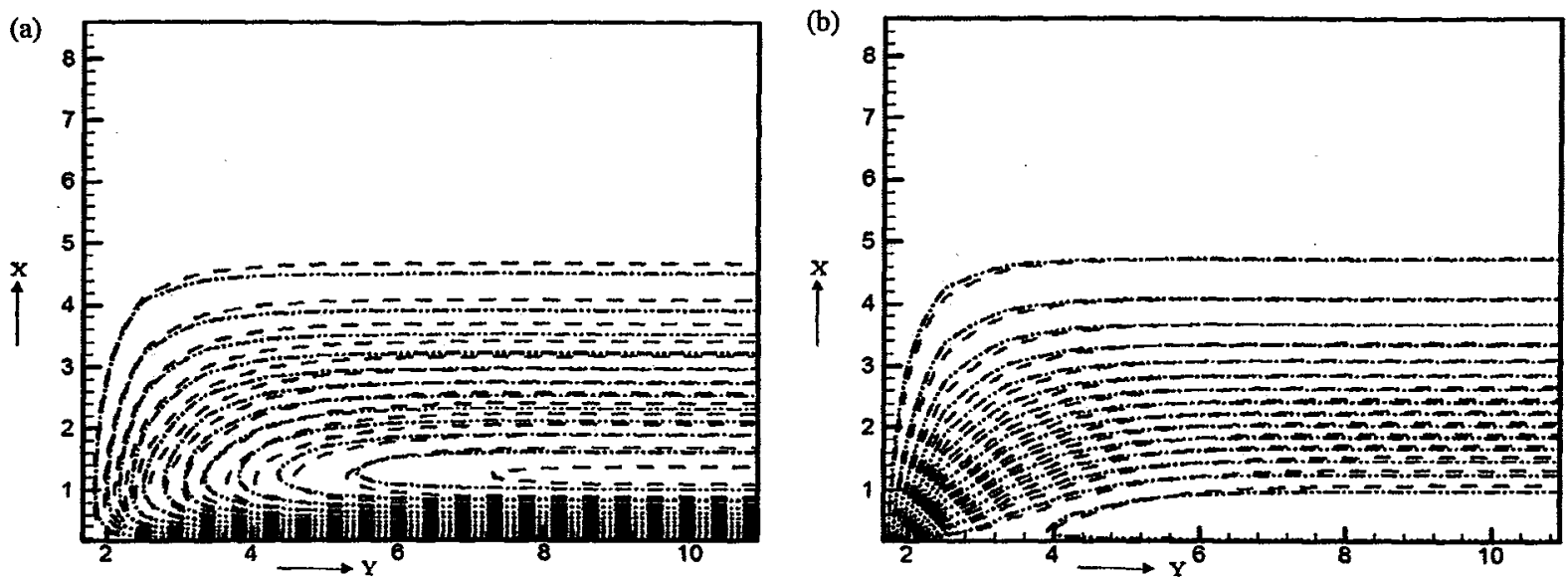

Fig. 7. Illustration of (a) streamlines for $G_{\mathrm{a}}=0.50$ and $G_{\mathrm{a}}=1.50$ and (b) isotherms for $G_{\mathrm{a}}=0.50$ and $G_{\mathrm{a}}=1.50$.

to $D_{\mathrm{u}}=1.0$ respectively. Because the thermal boundary layer thickness are increased due to increasing of that Dufour number $D_{\mathrm{u}}$. Generally, by increasing of Eckert number $E_{\mathrm{c}}$, the heat energy is gathered in the fluid by the drag force i.e., frictional force. In this reasons temperature field increases due to increasing Eckert number $E_{\mathrm{c}}$. On the other hand, Figure 5(a) carried out temperature profiles for differences values of radiation parameter $R_{\mathrm{a}}$. Physically, radiation parameter $R_{\mathrm{a}}$ provides more heat into the fluid, which leads to increases the thermal boundary layer thickness by increasing the values of radiation parameter $R_{\mathrm{a}}$. Also heat source parameter $S$ enhanced the temperature of the fluid field. The effects of chemical reaction parameter $\left(G_{\mathrm{a}}\right)$, Schmidt number $\left(S_{\mathrm{c}}\right)$ Soret number $\left(S_{\mathrm{r}}\right)$ and
Dufour number $\left(D_{\mathrm{u}}\right)$ on concentration profiles are displaced in Figure 5(b). From Figure 5(b), we have captitalized that concentration profiles decreased by $34.49 \%$, $25.50 \%, 24.25 \%$ and $16.95 \%$ for changes as $G_{\mathrm{a}}=5.0$ to $G_{\mathrm{a}}=1.0, S_{\mathrm{c}}=0.6$ to $S_{\mathrm{c}}=0.95, S_{\mathrm{r}}=1.0$ to $S_{\mathrm{r}}=2.0$ and $D_{\mathrm{u}}=$ 1.0 to $D_{\mathrm{u}}=2.0$ respectively.

The outcome of radiation parameter $R_{\mathrm{a}}$ on streamlines and isotherms are scrutinized in Figures 6(a) and (b). Here, we carried out that both momentum and thermal boundary layer thickness are increasing for the increasing of radiation parameter from $R_{\mathrm{a}}=0.50$ to $R_{\mathrm{a}}=1.50$. The impact of chemical reaction parameter $G_{\mathrm{a}}$ on streamlines and isotherms are showed in Figures 7(a) and (b). We capitalized that momentum boundary layer thickness

Table I. Variation of different parameters on skin friction coefficient, Nusselt number and Sherwood number.

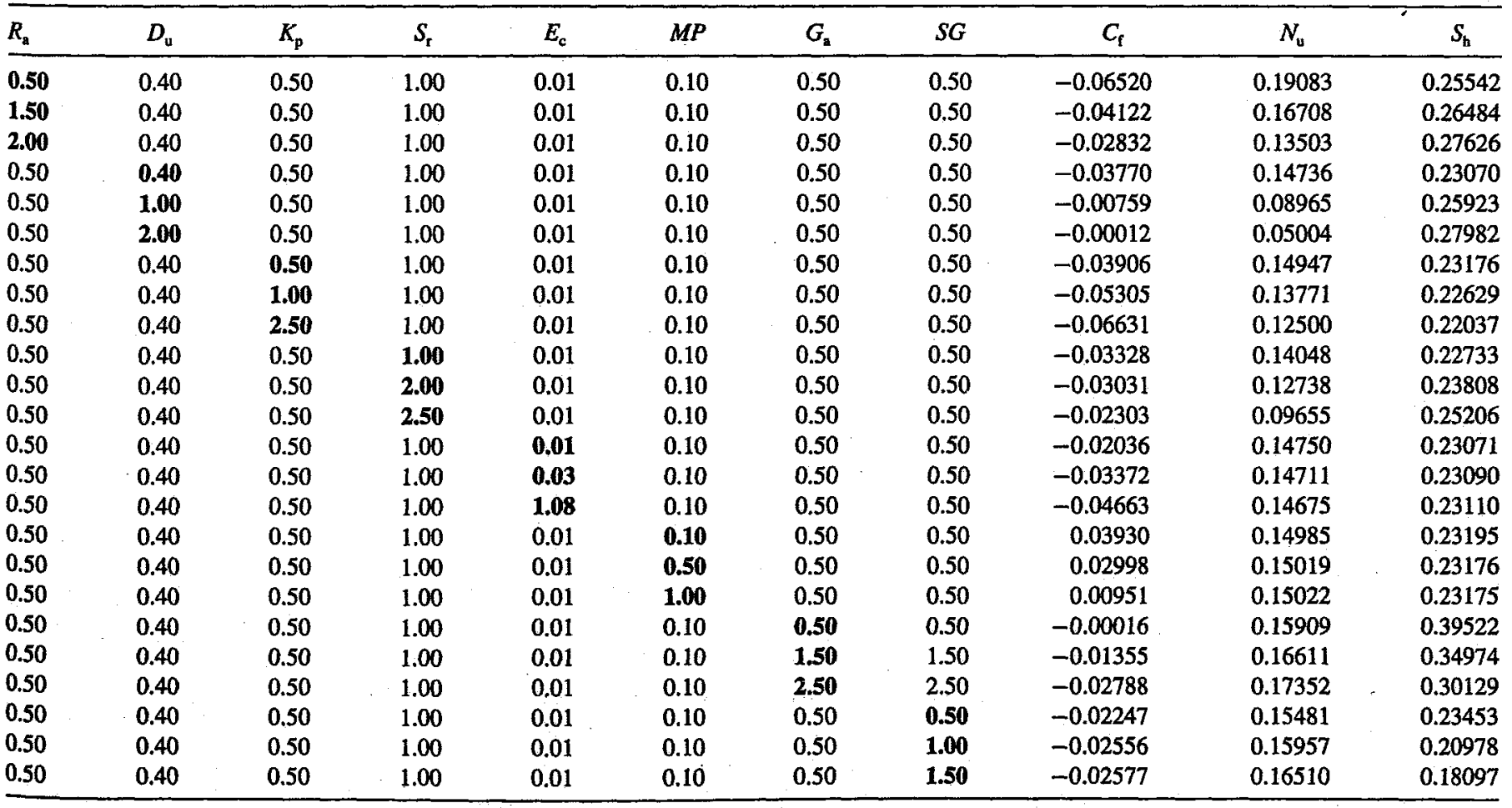


Table II. Comparison of the accuracy of the present results with the previous results by Haque et al. ${ }^{2}$

\begin{tabular}{|c|c|c|c|c|c|c|c|c|c|c|}
\hline \multirow[b]{2}{*}{$\begin{array}{l}\text { Increased } \\
\text { parameters }\end{array}$} & \multicolumn{5}{|c|}{$\begin{array}{c}\text { Previous results by } \\
\text { Haque et al. }\end{array}$} & \multicolumn{5}{|c|}{ Our present results } \\
\hline & $U$ & $\bar{T}$ & $\bar{C}$ & $\bar{\Gamma}$ & $\begin{array}{l}\text { Skin } \\
\text { fri. }\end{array}$ & $U$ & $\bar{T}$ & $\bar{C}$ & $\bar{\Gamma}$ & $\begin{array}{l}\text { Skin } \\
\text { fri. }\end{array}$ \\
\hline VP & & & & Inc & & & & & Inc & \\
\hline$M P$ & & & & Inc & Dec & & & & Inc & Dec \\
\hline$S G$ & & & & & & & & & & \\
\hline$S_{r}$ & & Dec & Inc & & Inc & & Inc & Dec & & \\
\hline$S c$ & Dec & & Dec & Inc & & Dec & & Dec & Inc & \\
\hline$K_{p}$ & Dec & & & Inc & Dec & Dec & & & Inc & Dec \\
\hline$D u$ & & Inc & Dec & Dec & Inc & & Inc & Dec & Dec & Inc \\
\hline$E_{\mathrm{c}}$ & Inc & Inc & & & & Inc & Inc & & & \\
\hline$P_{\mathrm{r}}$ & Dec & & & & & Dec & & & & \\
\hline
\end{tabular}

increases due to the increase of chemical reaction parameter from $G_{\mathrm{a}}=0.50$ to $G_{\mathrm{a}}=1.50$ but thermal boundary layer thickness decreases by the increasing of chemical reaction parameter from $G_{\mathrm{a}}=0.50$ to $G_{\mathrm{a}}=1.50$.

Table I represents the numerical values of different parameters such as radiation parameter $\left(R_{\mathrm{a}}\right)$, Dufour number $\left(D_{\mathrm{u}}\right)$, permeability of porous medium $\left(K_{\mathrm{p}}\right)$, Soret number $\left(S_{\mathrm{r}}\right)$, Eckert number $(E c)$, microrotational parameter $(M P)$, chemical reaction parameter $\left(G_{\mathrm{a}}\right)$ and spin gradient viscosity parameter $(S G)$ on skin friction $\left(C_{f}\right)$, Nusselt number $\left(N_{\mathrm{u}}\right)$ and Sherwood number $\left(S_{\mathrm{h}}\right)$ for Micropolar fluid. It is showed that the skin friction coefficient increases with the enhances of radiation parameter, Dufour number and Soret number but decreases qualitatively with an increase in Eckert number, microrotational parameter, permeability of porous medium, chemical reaction parameter and spin gradient viscosity parameter. Physically, skin friction which is increased the rate of change of velocity in the fluid flow. On the other hand the Nusselt number is an increasing functions of radiation parameter, Dufour number, permeability of porous medium, Soret number and Eckert number, whereas this tendency is quite opposite for chemical reaction parameter, microrotational parameter and spin gradient viscosity parameter. Generally, convective heat transfer is produced by the increase of radiation parameter, Dufour number, permeability of porous medium, Soret number and Eckert number which increase the Nusselt number. Also it is noticed that the Sherwood number increases with the increasing of radiation parameter, Dufour number, Eckert number and Soret number but decreases qualitatively with the increasing of in microrotational parameter, chemical reaction parameter, permeability of porous medium and spin gradient viscosity parameter. Generally, convective mass transfer is increased with the increase of Sherwood number but when it is decreased then the opposite observations are happens. Also, from Table II we have observed that, all the qualitative behaviours are same with the results of Haque et al. ${ }^{2}$ without just only for few results. It has been seen that Soret number $S_{\mathrm{r}}$ increases temperature profiles and decrease concentration profiles in our problems but opposite at the work of Haque et al. ${ }^{2}$

\section{CONCLUSIONS}

The following conclusions have been drawn by the above present numerical investigation,

(1) The skin friction coefficient increases with radiation parameter, Dufour number, permeability of porous medium, Soret number and microrotational parameter but decreases qualitatively with an increase in Eckert number, chemical reaction parameter and spin gradient viscosity parameter.

(2) It is observed that angular velocity profiles increases for the increasing of Grashof number, modified Grashof number and heat source parameter but decreasing due to increase of permeability of porous medium and Schmidt number.

(3) Velocity profiles are increasing respectively as Grashof number, modified Grashof number and heat source parameter.

(4) It is noticed that the Sherwood number increases with the increasing of radiation parameter, Dufour number, Eckert number and Soret number but decreases qualitatively with the increasing of in microrotational parameter, chemical reaction parameter, permeability of porous medium and spin gradient viscosity parameter.

(5) In view of that chemical reaction parameter, Schmidt number, Soret number and Dufour number decreases the concentration profiles.

\section{NOMENCLATURE}

$x, y$ Cartesian coordinates (m)

$u, v$ Velocity components $\left(\mathrm{ms}^{-1}\right)$

$U$ Dimensionless primary velocity $\left(\mathrm{ms}^{-1}\right)$

$\bar{\Gamma}$ Dimensionless angular velocity $\left(\mathrm{rads}^{-1}\right)$

$\bar{T}$ Dimensionless fluid temperature (K)

$\bar{C}$ Dimensionless fluid concentration (-)

$S G \quad$ Spin gradient viscosity parameter (-)

$G_{\mathrm{a}}$ Chemical reaction parameter (-)

$V P \quad$ Vortex viscosity parameter (-)

$C_{\mathrm{f}}$ Skin friction (-)

$S_{\mathrm{h}}$ Sherwood number (-)

$D_{\mathrm{u}}$ Dufour Number (-)

$P_{\mathrm{r}}$ Prandlt number (-)

$E_{\mathrm{c}}$ Eckert number (-)

$G_{\mathrm{r}}$ Grashof number (-)

$G_{\mathrm{m}} \quad$ Modified Grashof number (-)

$R_{\mathrm{a}}$ Radiation parameter (-)

$S$ Heat source parameter (-)

$K_{\mathrm{p}}$ Permeability of porous medium (-)

$M P$ Microrotational parameter (-)

$S_{\mathrm{r}}$ Soret number (-)

$N_{\mathrm{u}}$. Nusselt number $(-)$

$S_{\mathrm{c}} \quad$ Schmidt number $(-)$ 


\section{References and Notes}

1. A. C. Eringen, Journal of Mathematics and Mechanics 16, 1 (1966).

2. M. Z. Haque, M. M. Alam, M. Ferdows, and A. Postelnicu, Journal of King Saud University Engineering Sciences 24, 71 (2012).

3. G. Ahmadi, International Journal of Engineering and Science 14, 639 (1976).

4. T. Ariman, M. A. Turk, and N. D. Sylvester, International Journal of Engineering and Science 12, 273 (1974).

5. M. K. Nayak, AMSE Journals Series: Modelling $B$ 84, 52 (2015).

6. M. Balram and V. U. K. Sastry, Int. J. Heat Mass Transfer 16, 437 (1973).

7. F. S. Lien, T. M. Chen, and C. K. Chen, J. Heat Transfer 112, 504 (1990).

8. R. S. Tripathy, G. C. Dash, S. R. Mishra, and M. M. Hoque, Engineering Science and Technology, an International Journal 19, 1573 (2016).

9. N. A. Khan, S. Khan, and A. Ara, Propulsion and Power Research 6,285 (2017).

10. D. H. Doh and M. Muthtamilselvan, International Journal of Mechanical Sciences 130, 350 (2017).

11. T. J. Muhammad and A. Siddiqui, J. Mol. Liq. 249, 831 (2018).

12. P. John, Recent Advanced Engineering and Science 5, 405 (1970).

13. M. G. Reddy and N. B. Reddy, Journal of Applied Fluid Mechanics 4, 7 (2011).

14. E. M. Sparrow and R. D. Cess, International Journal Heat and Mass Transfer 3, 267 (1961).

15. P. Ganesan and G. Palani, Heat Mass Transfer 21, 277 (2003).

16. N. Islam and M. M. Alam, Journal of Naval Architecture and Marine Engineering 4, 43 (2007).

17. P. Ganesan and G. Palani, International Journal of Heat Mass Transfer 47, 4449 (2004).

18. M. Bhuvaneswari, S. Sivasankaran, and Y. J. Kim, World Applied Journal 10, 774 (2010).
19. B. Mohanty, S. R. Mishra, and H. B. Pattanayak, Alexandria Engineering Journal 54, 223 (2015).

20. S. R. Mishra, S. Baag, and D. K. Mohapatra, Engineering Science and Technology, an International Journal 19, 1919 (2016).

21. S. Rawat, S. Kapoor, and R. Bhargava, Journal of Applied Fluid Mechanics 9, 321 (2016).

22. R. Biswas, M. Mondal, D. R. Sarkar, and S. F. Ahmmed, Journal of Advances in Mathematics and Computer Science 23, 1 (2017).

23. M. M. Rashidi, B. R. Navid, and F. S. Abbasbandy, Ain Shams Engineering Journal 5, 901 (2014).

24. R. Biswas and S. F. Ahmmed, J. Heat Transfer 140 (2018).

25. S. F. Ahmmed, R. Biswas, and M. Afikuzzaman, J. Nanofluids 7, 891 (2018).

26. B. Mahanthesh, B. J. Gireesha, and R. S. R. Gorla, Journal of the Association of Arab Universities for Basic and Applied Sciences 23, 75 (2017).

27. R. Ahmed, B. M. J. Rana, R. Uddin, M. M. Islam, and S. F. Ahmmed, Physical Science International Journal 12, 1 (2016).

28. M. Afikuzzaman, M. Ferdows, and M. M. Alam, Procedia Engineering 105, 287 (2015).

29. M. Afikuzzaman and M. M. Alam, Science and Technology Asia 21, 59 (2016).

30. G. S. Seth, M. K. Mishra, and R. Tripathi, Journal of the Brazilian Society of Mechanical Sciences and Engineering 40, 103 (2018).

31. S. Farooq, T. Hayat, B. Ahmad, and A. Alsaedi, Journal of the Brazilian Society of Mechanical Sciences and Engineering 40, 159 (2018).

32. R. Biswas, M. Mondal, S. F. Ahmmed, and M. Afikuzzaman, Frontiers in Heat and Mass Transfer (FHMT) 11, 13 (2018).

33. M. Kumari and S. Jain, J. Nanofluids 8, 938 (2018).

34. S. Jain and P. Gupta, J. Nanofluids 8, 929 (2018).

35. S. Muhammad, S. I. A. Shah, G. Ali, M. Ishaq, S. A. Hussain, and Z. Shah, J. Nanofluids 8, 957 (2018).

36. G. C. Shit and S. Mukherjee, J. Nanofluids 8, 998 (2018). 